\title{
El pensamiento crítico como un paso adelante en la Formación Profesional. Estudiantes de Formación Profesional inmersos en centros de alta tecnología.
}

\author{
Concha Solano Martínez ${ }^{\mathrm{a}}$, Sandra Clara Trujillo ${ }^{\mathrm{b}}$, María Teresa Guillot-Ferriols ${ }^{\mathrm{b}}$, Luis \\ Gómez Estrada ${ }^{a}$, José Luis Gómez Ribelles ${ }^{\text {b,c }}$ \\ aIkasia Technologies SL, Valencia info@ikasia.es \\ ${ }^{\text {b} C e n t r o ~ d e ~ B i o m a t e r i a l e s ~ e ~ I n g e n i e r i ́ a ~ T i s u l a r, ~ U n i v e r s i t a t ~ P o l i t e ̀ c n i c a ~ d e ~ V a l e ̀ n c i a, ~ j l g o m e z @, t e r . u p v . e s ~}$ \\ ${ }^{\mathrm{c} C e n t r o ~ d e ~ I n v e s t i g a c i o ́ n ~ B i o m e ́ d i c a ~ e n ~ R e d: ~ B i o i n g e n i e r i ́ a, ~ B i o m a t e r i a l e s ~ y ~ N a n o m e d i c i n a, ~ V a l e n c i a ~}$
}

\begin{abstract}
This communication summarizes the results of the Erasmus + project "CRITICAL THINKING AS A STEP FORWARD IN VET EDUCATION: VET students immersed in high technology teams" in which european technologybased companies, higher research centers and vocational training centers have participated. For two years, strategies have been designed to develop training in critical thinking tools in vocational students during internships in high-tech centers. We have chosen a reduced set of critical thinking skills that we think can act as a catalyst for student training throughout their professional careers, and in creative work that contributes effectively to the company's objectives. The debate has crystallized in the writing of two tools: a template of help to the tutor in the company for the preparation of the work plan of the student and another template for the student's laboratory notebook. In one case and in another the writing of these notebooks requires the student and the tutor to reflect on the critical thinking skills that are intended to develop in the student.
\end{abstract}

Keywords: critical thinking, VET, technology companies, research centres

\section{Resumen}

Esta comunicación resume parte de los resultados del proyecto Erasmus+ "CRITICAL THINKING AS A STEP FORWARD IN VET EDUCATION: VET students immersed in high technology teams" en el que han participado empresas de base tecnológica, centros superiores de investigación y centros de formación profesional europeos. Durante dos años se han diseñado estrategias para desarrollar la formación en herramientas de pensamiento crítico en los estudiantes de formación profesional durante prácticas realizadas en centros de alta tecnología. Se ha elegido un conjunto reducido de capacidades de pensamiento crítico que pensamos que pueden actuar de desencadenante de la formación del estudiante a lo largo de toda su carrera profesional, y en un trabajo creativo que contribuya de forma efectiva a los objetivos de la empresa. El debate ha cristalizado en la redacción de dos herramientas: una plantilla 
de ayuda al tutor en la empresa para la preparación del plan de trabajo del estudiante y otra plantilla para el cuaderno de laboratorio del estudiante. En un caso y en otro la redacción de estos cuadernos obliga al estudiante y al tutor a una reflexión relacionada con las capacidades de pensamiento crítico que se pretenden desarrollar en el estudiante.

Palabras clave: pensamiento crítico, formación profesional, empresas de base tecnológica, centros de investigación.

\section{Introducción.}

Desde hace al menos tres décadas y cada vez con más intensidad los organismos internacionales responsables de la educación y el desarrollo científico e industrial ponen de manifiesto la necesidad de analizar a fondo las capacidades que deben formarse en los profesionales para que estén en disposición de contribuir al desarrollo de una tecnología que avanza y cambia de forma vertiginosa. Los resultados de estos estudios pueden diferenciarse en los matices, pero son absolutamente coincidentes en el fondo. La formación de los profesionales a cualquier nivel, desde la enseñanza secundaria, debe enfocarse fundamentalmente a una serie de elementos que podríamos llamar transversales. En el informe final de la Comisión Internacional para la educación en el siglo XXI, coordinado por Jaques Delors se clasifican en cuatro pilares que llamaron "Aprender a aprender", "Aprender a ser", "Aprender a hacer" y "Vivir juntos", que se traducen en el desarrollo de herramientas personales como la flexibilidad, iniciativa, responsabilidad, asunción de riesgos, creatividad, herramientas sociales como la empatía, el trabajo en grupo, capacidad de establecer relaciones y herramientas de aprendizaje como organización, herramientas metacognitivas, respuesta a un fallo y salir adelante después de un fallo (Delors, 1996). En el documento de trabajo de la UNESCO “ The futures of learning 2: What kind of learning for the 21st century?, Cynthia Luna Scott desarrolla en detalle las competencias en las que habría que enfocar la educación para formar las personas que se han de incorporar al desarrollo tecnológico de este siglo, ya avanzado (Scott, 2015).

Aunque este mensaje llega a los gestores, profesores y estudiantes de la formación profesional, los sistemas educativos tienen una gran inercia y los cambios educativos de fondo son siempre más lentos que los cambios que se producen en la sociedad.

La visión del proyecto CRITICAL THINKING AS A STEP FORWARD IN VET EDUCATION: $V E T$ students immersed in high technology teams se basa en la idea de que se puede contribuir de forma muy significativa a la formación de los profesionales en las capacidades que se requieren para involucrarse en empresas y proyectos tecnológicos a base de trabajar en el desarrollo del pensamiento crítico, es decir, con estrategias que forman al estudiante o al profesional en "pensar bien". Esta contribución a la formación puede hacerse de forma muy ilusionante cuando se realiza involucrando al estudiante en una empresa de desarrollo tecnológico. 
Cómo organizar el trabajo de un estudiante VET en la empresa de forma que se estimulen sus capacidades de pensamiento crítico y su creatividad no es algo trivial. Posiblemente no es algo intuitivo para los gestores y los componentes de los equipos de trabajo de estas empresas incluso aunque sean profesionales con probadas capacidades de pensamiento crítico. Hay un camino largo desde comprender y saber hacer algo hasta saberlo enseñar. En este proyecto, gracias al programa Erasmus + ha sido posible reunir a un colectivo importante de personas procedentes de empresas tecnológicas, centros de investigación y centros de formación VET. Personas interesadas en aportar ideas y estrategias sobre la formación de los profesionales en el centro de formación VET y en la empresa.

\section{Objetivos.}

Es extraordinariamente difícil diseñar estrategias formativas para desarrollar en un programa de estudios a cualquier nivel cada una de las competencias identificadas como necesarias para los profesionales de este siglo. Es aún más difícil pensar en precisar actividades concretas en las prácticas de un estudiante de formación profesional en una empresa tecnológica para contribuir a su formación en todas estas capacidades. Nuestra visión se centra, no en una propuesta tan detallada, si no en desarrollar ciertas competencias que tengan la capacidad de actuar como desencadenante de una actitud y una forma de pensar en el estudiante que le lleven a asumir la importancia de poner en práctica todas las demás. Nuestra propuesta es que la clave está en el desarrollo del pensamiento crítico. Estamos convencidos de que la formación en habilidades del Pensamiento Crítico puede ser un catalizador para disparar el proceso de adquirir las capacidades, herramientas y habilidades que se requieren a los profesionales del siglo XXI.

El objetivo del proyeto Erasmus+ $\mathrm{K} 2$ CRITICAL THINKING AS A STEP FORWARD IN VET EDUCATION: VET students immersed in high technology teams ha sido la elaboración de dos guías didácticas destinadas a facilitar la preparación de prácticas de estudiantes de Formación Profesional en empresas tecnológicas, sobre la bvase del pensamiento crítico.

O1.- METHODOLOGICAL GUIDE FOR HIGH TECH CENTERS TO HOST VET STUDENT INTERNSHIPS.

O2.- CURRICULAR DEVELOPMENT FOR QUALITY AND INNOVATION IN VET CENTERS THROUGH CROSS-SECTORAL CO-OPERATION.

\section{Desarrollo de la innovación}

La metodología de trabajo para el desarrollo de los materiales didácticos objetivo de este proyecto ha buscado situarse en el punto de vista de un graduado que inicia su trabajo profesional en la empresa e identificar y poner énfasis en los aspectos que demuestran que la clave del desarrollo personal y profesional está en el pensamiento crítico. También se ha cuidado la forma de exponer los resultados de este análisis con un lenguaje que sea próximo 
al estudiante, aunque ello pueda hacernos incurrir en imprecisiones al definir algunos términos. El trabajo parte, por lo tanto, de constatar una serie de rasgos de cómo es la actividad cotidiana en un equipo de trabajo de una empresa de base tecnológica o en un grupo de investigación. Se revelan determinadas características, por ejemplo:

- Los objetivos de investigación se definen con precisión.

- Se establece con claridad a dónde se quiere ir.

- Se establecen hipótesis realistas, basadas en argumentos muy bien analizados.

- Continuamente surgen nuevas necesidades que requieren aprender nuevos conceptos y técnicas.

- La formación más valorada en el profesional es aquélla que posibilita aprender por uno mismo a lo largo de toda la carrera profesional.

- Hay que saber expresar con claridad en una reunión las propias opiniones, incluso si contradicen a las de otros.

- Uno debe ser capaz de juzgar si aquello que ha escrito lo va a poder entender otro.

- Ser capaz de ponerse en el lugar de un lector que no ha estado involucrado en la redacción.

- Es necesario analizar de forma crítica la información que se obtiene de cualquier fuente.

- No todo lo que dice un libro o una página web es cierto, ni todo lo que dice una fuente que en general es fiable está libre de errores.

- Contribuir al trabajo del grupo es más que realizar unas tareas programadas. Es importante valorar el resultado desde la experiencia de cada uno.

A partir de aquí se ha seleccionado o definido unas habilidades de pensamiento crítico sobre las que poner el foco en este estudio y se ha debatido sobre estrategias para desarrollarlas en los estudiantes, por una parte en los propios centros de formación profesional, de forma previa a la realización de las prácticas y por otra en la propia empresa.

En relación con la formación en el centro educativo se ha preparado un desarrollo curricular que aprovecha la enseñanza en lengua extranjera. Se ha preparado tres unidades didácticas que desarrollan los objetivos lingüisticos trabajando con materiales relacionados con el pensamiento crítico y con las nuevas tecnologías. Esta línea de trabajo ha tenido como resultado el documento CURRICULAR DEVELOPMENT FOR QUALITY AND INNOVATION IN VET CENTERS THROUGH CROSS-SECTORAL CO-OPERATION. Curricula Development in English subjects of VET syllabus, que puede encontrarse en la página web del proyecto (http://www.ikasia.es/es/proyecto-e/). Los contenidos lingüísticos de estas unidades son los que establece el Marco Común Europeo, según el cual el alumnado de grado medio de formación profesional debería de progresar de un nivel A2 adquirido en la secundaria o formación profesional inicial hacia un B1 una vez finalizados los estudios del grado. No nos extenderemos más sobre este aspecto del proyecto en esta comunicación por limitación de espacio.

\section{Resultados}


En relación con la preparación del estudiante en la empresa se ha desarrollado la guía: METHODOLOGICAL GUIDE FOR HIGH TECH CENTERS TO HOST VET STUDENT INTERNSHIPS (http://www.ikasia.es/es/proyecto-e/). Esta guía se inicia con unas orientaciones generales, de aplicación en un abanico amplio de situaciones o empresas tecnológicas, para la integración de estudiantes o graduados de formación profesional. Posteriormente, se presenta una plantilla del cuaderno de laboratorio como herramienta esencial para guiar la reflexión del estudiante sobre los distintos aspectos de su trabajo en la empresa y un docuemnto de ayuda a su tutor en la empresa para preparar su plan de trabajo de forma que estimule la formación de capacidades de pensamiento crítico. Exponemos el contenido esencial en los siguientes apartados.

\subsection{Capacidades de pensamiento crítico en estudiantes de formación profesional.}

Está fuera del objetivo de esta comunicación recoger las muchas definiciones que se han dado en la literatura sobre el pensamiento crítico y los estudios teóricos sobre las características, disposiciones y habilidades que se suponen a un pensador crítico. Nos conformaremos con intentar dar una idea sucinta de las capacidades de pensamiento crítico sobre las que el proyecto se ha centrado aún con el riesgo de no ser suficientemente precisos. El lector puede dirigirse a la amplia lilteratura existente sobre el pensamiento critico para contrastar nuestras opciones (ver por ejemplo, Pithers, 1999; Ennis, 2011; Lopez Aymes, 2012; Paul y Elder, 2005)

C1.- Evaluar la propia forma de razonar.

Probablemente es el punto clave del pensamiento crítico y al que se dirige todo lo demás. Un profesional no debería aceptar ninguna idea, ningún procedimiento de trabajo o de producción, ningún resultado experimental sin haberlo valorado usando su razonamiento lógico y haber llegado a través de la razón a convencerse de que lo que se está haciendo es correcto, o lo que se está aceptando es verdad. Esto no es un proceso natural en ninguna persona y tampoco en un estudiante o profesional recién titulado. Al iniciarse en la experiencia laboral, puede que la tendencia sea más bien la de pensar que uno debe aceptar su desconocimiento sobre el mundo de la empresa y no está para dar lecciones a nadie, asumir que si las cosas se hacen así es porque se tienen que hacer así y es mejor no tratar de criticarlas. La actividad en la empresa durante la práctica del estudiante tendría que empezar por convencer a ese profesional de que precisamente de cara a su formación y a aprender los modos de trabajo, es esencial desde el primer momento no aceptar nada de lo que no se haya convencido él mismo gracias a su razonamiento, capacidad de informarse y entender la información que recibe, capacidad de hacer preguntas clarificadoras y las demás capacidades de pensamiento crítico que enunciamos a continuación. En consecuencia, el estudiante debería:

- Estar convencido de la necesidad de comprender a fondo cada tema con el que se encuentre y dedicar a ello el tiempo y esfuerzo necesario antes de iniciar una acción o tomar una decisión.

- Estar convencido de que la crítica a un procedimiento o idea establecida puede ser una forma valiosa de aprender siempre que se esté dispuesto a aceptar estar equivocado. 
- En este sentido, tomar en consideración de forma abierta las opiniones de los demás y estár en disposición de cambiar las propias ideas en función de esas opiniones.

- También en este sentido, aceptar las críticas de los demás y estar en disposición de debatirlas y en su caso cambiar en consecuencia.

- Verificar la relevancia, profundidad y amplitud del pensamiento (asegurarse de que se extiende el razonamiento a toda la complejidad del asunto en cuestión, que no se pasa por alto ningún aspecto o ninguna información disponible, que se ha tenido en cuenta con todos los puntos de vista)

C2.- Plantear objetivos. Plantear problemas.

El pensamiento crítico ha de ser un pensamiento activo, dirigido a analizar un razonamiento o tomar una decisión y el primer paso para ello es asegurarse que se comprende con claridad lo que se está analizando y se formula de forma concreta. El pensamiento crítico plantea esta acción en términos de formulación de un problema (entendido de forma genérica).

La formación del estudiante debería llevarle a:

- Explicar con sus propias palabras el objetivo de la tarea que tiene encomendada: ensayos experimentales, cálculos, documentación...

- Explicar con sus propias palabras el problema que se le plantea y sobre el que necesita reflexionar

- Plantearse objetivos o tareas secundarias que considera necesarias para llegar al objetivo final.

C3.- Plantear preguntas relevantes.

Las estrategias de formación del pensamiento crítico del estudiante en el centro educativo incluyen como una de las herramientas importantes la formulación de preguntas. Las preguntas ayudan a impulsar el pensamiento reflexivo, a ampliar las destrezas del pensamiento, clarificar la comprensión, enlazar distintas ideas, fomentar la curiosidad, plantear retos (Lopez Aymes, 2012). Del mismo modo una de las claves para aprender a aprender es aprender a formular preguntas, el propio planteamiento de la pregunta exige una reflexión crítica sobre lo que es importante para avanzar en la comprensión de un tema, juzgar la validez de una información, indagar sobre la existencia de conflictos de interés.

En este sentido, la formación del estudiante debe guiarle hacia la formulación de las preguntas importantes en cada ámbito y más en concreto:

- Expresar con sus propias palabras la pregunta en cuestión.

- Formular una cuestión de distintas formas alternativas para llegar a concretarla y hacerse comprender lo más posible.

- Distinguir las preguntas importantes de las triviales.

- Distinguir las preguntas a las que es capaz de resolver de las que no están a su alcance. 


\section{C4.- Plantear hipótesis.}

Uno de los elementos esenciales del método científico es el planteamiento de una hipótesis que luego es contrastada o no con la experiencia. También es un elemento clave en el análisis de los resultados de una tarea profesional. El planteamiento de una hipótesis que anticipa el resultado de un ensayo o una tarea obliga a la reflexión sobre los fundamentos que la gobiernan y posteriormente el contraste entre la hipótesis y los resultados obtenidos estimula la reflexión sobre los posibles errores en la realización de los procedimientos o en los conocimientos que se tenían sobre el tema.

- El profesional debe convencerse de la importancia de reflexionar sobre cualquier ensayo a realizar para anticipar su resultado, utilizando para ello los conocimientos que tenga.

- El estudiante debe valorar los conocimientos adquiridos previamente que guarden relación con lo nuevo que va a aprender en la empresa y contrastarlos con lo que aprende de nuevo

- Formular hipótesis precisas, que realmente anticipen el resultado que se espera obtener.

- Formular una hipótesis previa a la búsqueda de información sobre un tema.

\section{C5.- Aprender a aprender, buscar información}

Aprender por uno mismo a lo largo de toda la carrera profesional requiere desarrollar determinadas capacidades. En la actualidad, la búsqueda de información sobre un tema concreto puede sumergir al profesional en una montaña de documentos de todo tipo y origen. Es necesario aprender a seleccionar de entre ellos aquellos que merecen cierta confianza, los que a priori merecen emplear el tiempo necesario para leerlos y valorarlos, y luego a valorar la credibilidad de la información obtenida contrastándola con la que llega de distintas fuentes y empleando el propio razonamiento. No es algo fácil y necesita de una aproximación guiada. Los educadores y los miembros del equipo de la empresa pueden hacer una labor importante en la formación de esta capacidad en los estudiantes, para que lleguen a:

- Expresar con claridad y con sus propias palabras la información obtenida

- Distinguir entre la información relevante de la irrelevante

- Recoger tanto las informaciones que están a favor de la propia opinión o de la hipótesis formulada como las que las contradicen.

- Sacar conclusiones de la información recibida sólo cuando esa información está apoyada por hechos y por un razonamiento lógico, descartar información no fundamentada.

- Distinguir entre la información en sí y las inferencias que uno mismo u otros hacen de esa información.

\section{C6.- Tener una mentalidad abierta.}

Recogemos aquí una serie de actitudes que se han resaltado como disposiciones del pensador crítico: humildad intelectual (escuchar y aceptar la opinión de otros, reconocer errores), coraje intelectual (disposición a defender las propias ideas), empatía intelectual (capacidad para 
ponerse en el lugar de otro para comprender su punto de vista). Además de disposiciones necesarias para el pensamiento crítico lo son también para el trabajo en grupo.

- Ser capaz de descubrir y admitir sus propias equivocaciones, errores o ideas falsas

- No juzgar sobre aquello que se desconoce

- Distinguir lo que se comprende de un tema y lo que no se conoce suficientemente.

- Evaluar tanto las ideas que son populares en su grupo o en su entorno en general como las que no lo son.

- No temer el rechazo de los demás por defender una idea propia

- Admitir en los debates que uno puede estar equivocado y expresarlo de ese modo

- $\quad$ Situarse en el lugar de los demás.

\section{C7.- Tener integridad intelectual.}

La honestidad en el trabajo en una empresa es un aspecto de enorme importancia. Por ejemplo, la trascendencia de ocultar al grupo determinada información que va en contra de tus ideas o esconder un error cometido puede llevar a la toma de decisiones equivocadas en el equipo que pueden ser muy costosas. También el hecho de emplear falacias para dirigir los debates hacia el propio beneficio intelectual o material o para situarse sobre otras personas del grupo, para "ganar" la discusión, puede tener efectos graves sobre el trabajo y las relaciones personales en el seno del grupo. En este sentido es necesario reforzar determinadas disposiciones:

- Identificar y reconocer las inconsistencias dentro del propio pensamiento

- Identificar y reconocer las inconsistencias entre las propias ideas y la forma de actuar

- $\mathrm{Al}$ defender las propias ideas identificar aquello que busca la objetividad y lo que busca el propio beneficio.

C8.- Expresarse bien, de forma efectiva, tanto oralmente como por escrito.

La efectividad en la comunicación oral y escrita es una de las herramientas básicas del trabajo en grupo, pero es también un signo de la profundidad del propio razonamiento. La capacidad de expresarse con claridad es un signo de la claridad y seguridad en las propias ideas. Al mismo tiempo la expresión y la defensa de las propias conclusiones son un medio efectivo de ayuda al pensamiento crítico, obligan a precisar el propio pensamiento y descubren los puntos de inconsistencia o lo que es vago, o impreciso o no se comprende bien. La formación en este aspecto vendrá de la práctica y la crítica de uno mismo y de otros.

- El estudiante debe poder juzgar por sí mismo si aquello que expresa de forma oral o por escrito es comprensible para los demás.

- Detectar si en su discurso se omite información sin la cual no es posible comprender el mensaje.

- Detectar y reconocer si lo que se enuncia resulta vago o impreciso porque realmente uno mismo no ha llegado a conclusiones más precisas. 
- Organizar el contenido de un texto resaltando las ideas más relevantes y separándolas de lo que puede ser información accesoria.

\section{C9.- Ser perseverante.}

La vida profesional obliga a enfrentarse a multitud de nuevos conceptos, conocimientos fundamentales, técnicas, procedimiento de uso de equipamientos, trabajo en equipo, trabajo con objetivos y plazos prefijados. No es fácil, es necesaria una gran perseverancia. Adquirir las habilidades del pensamiento crítico ayuda a alcanzar las competencias necesarias para realizar todas esas actividades y disfrutar de ellas,aunque estas competencias no sean sencillas de adquirir. Hablar de perseverancia es también hablar de optimismo, de autoestima y confianza en la capacidad de superar todos los obstáculos. Como disposiciones concretas en este campo habría que lograr que el estudiante:

- $\quad$ No se dé por vencido ante una tarea o un problema complejos

- Sea capaz de descomponer una tarea compleja en otras más accesibles para él.

- Destine la cantidad de tiempo adecuada a la resolución de un problema o a la comprensión de un tema.

\subsection{El cuaderno de laboratorio}

El cuaderno de laboratorio es una de las herramientas esenciales del trabajo experimental en cualquier laboratorio. Se escribe en forma de diario, y en muchos laboratorios se exige la firma del autor página a página e incluso la de un supervisor. Tradicionalmente se le ha dado gran relevancia no sólo como soporte donde se recopilan con detalle las metodologías y protocolos experimentales y se recogen los datos obtenidos, sino también como testimonio de la prioridad de los resultados e invenciones. El cuaderno avala la autoría de los ensayos. Los protocolos experimentales se escriben con suficiente detalle para que puedan ser reproducidos por otra persona, con ello constituyen una gran herramienta de transmisión de la experiencia dentro de la empresa o del grupo de investigación.

En este caso proponemos utilizar el cuaderno de laboratorio como herramienta de formación de los estudiantes VET en las competencias del pensamiento crítico. Proponemos una plantilla para el cuaderno de laboratorio especialmente pensado para los estudiantes VET que realicen prácticas en la empresa. Las entradas en el cuaderno de laboratorio contienen elementos que obligan a la reflexión crítica sobre el trabajo que se está realizando y sobre la medida en la que el estudiante ha comprendido la tarea que tiene asignada y su papel en el conjunto del trabajo. En particular en el cuaderno de laboratorio se deben escribir, con las propias palabras del estudiante, textos que muestren brevemente los siguientes aspectos:

- Comprensión del objetivo global del estudio, tal como se lo hayan planteado sus supervisores o como resulten de reuniones de trabajo en las que el propio estudiante participa.

- $\quad$ Las hipótesis del estudio. 
- La tarea concreta que se le ha asignado y a la que se refiere la entrada concreta que está anotando en el cuaderno de laboratorio ese día.

- Las razones que llevan a aplicar la metodología experimental que se le ha propuesto.

- Si se está realizando un ensayo experimental, una estimación inicial que anticipe cuál es el resultado que se espera obtener, es decir, una hipótesis sobre el ensayo concreto a realizar.

- Descripción del ensayo realizado y de los resultados obtenidos.

- Un juicio sobre la medida en que el resultado obtenido coincide razonablemente con la hipótesis planteada y las razones que explicarían que no fuera así.

- Un juicio sobre la reproducibilidad y/o el grado de subjetividad del ensayo realizado.

- Un juicio sobre la pertinencia de repetir el ensayo o darlo por bueno.

- Un resumen de la presentación de los resultados y los comentarios que hayan suscitado en otros miembros del grupo.

Este tipo de estructura tiene aspectos específicos para la formación del estudiante en pensamiento crítico. Probablemente no coincidirá completamente con la estructura del cuaderno de laboratorio de los técnicos de la plantilla de la empresa o de los investigadores experimentados, pero las diferencias no son de fondo, sino que se derivan de la concreción de las actividades encargadas a unos y a otros. En unos casos pueden ser tareas muy específicas, como un ensayo para determinar la densidad de un material sólido o de un fundido, y en otros casos puede ser más amplio como un experimento de cultivo celular o el diseño de un proceso de producción de una pieza concreta.

El hecho de que sistemáticamente, para cada experimento o actividad realizada, se requiera completar esta información obliga a desarrollar herramientas que claramente encajan dentro de las competencias del pensamiento crítico que van a ayudar a desarrollar .

\subsection{El cuaderno del tutor}

Las páginas siguientes recogen nuestra propuesta de una plantilla para ayudar al tutor de un estudiante en prácticas a preparar el plan de trabajo enfocado al desarrollo de las competencias de pensamiento crítico que hemos expuesto en el apartado 4.1, también para poder organizar el seguimiento del progreso del estudiante. Como se puede ver en el propio cuaderno, se propone empezar por presentarle al estudiante el trabajo en su conjunto, para hacerle comprender los objetivos, hipótesis y lo que se espera de su contribución a la consecución de los objetivos globales del proyecto. Como en todo el desarrollo de la práctica uno de los aspectos fundamentales es que sea el estudiante el que con sus propias palabras anote en su cuaderno de laboratorio estos aspectos, pidiendo las aclaraciones que necesite al propio tutor o a otros componentes del grupo.

El siguiente paso sería encargar al estudiante una tarea lo más concreta posible, que recoja una parte experimental que pueda llevarse a cabo en un periodo de tiempo reducido, quizá del orden de 10 a 15 días. Se pretende con ello evaluar las habilidades del estudiante en 
relación con determinados aspectos del pensamiento crítico, pero sobre todo orientarle desde el primer momento en un modo de trabajar reflexivo. En el cuaderno se sugieren distintos componentes que deberían incluirse en la tarea, como pueden ser la búsqueda de determinada información concreta, la comprensión de un protocolo experimental, la presentación de datos experimentales, la participación en reuniones de grupo y la forma en que recoge todo ello en su cuaderno de laboratorio.

Analizar con el estudiante después de esta primera tarea los resultados obtenidos, valorar sus puntos fuertes y ayudarle a orientar la forma en que se pueden mejorar los distintos aspectos tratados puede ser muy eficaz de cara al posterior desarrollo de la práctica.

A continuación, se propone que el tutor tome ciertas notas de la reorientación del plan de trabajo en la medida que sea pertinente o de la evolución del estudiante en relación con las competencias de pensamiento crítico, pero siempre sobre la base de que es el estudiante quien recoge en su cuaderno todo lo relacionado con la descripción de las tereas, los objetivos, hipótesis, resultados y valoraciones.

\section{Conclusiones}

El trabajo ha permitido diseñar herramientas prácticas para la integración de estudiantes o graduados de formación profesional en empresas de base tecnológica. Estas herramientas parten del convencimiento de que la formación en determinadas habilidades de pensamiento crítico en el profesional puede actuar como catalizador que evidencie el interés en continuar desarrollándolas durante toda la vida profesional y como herramienta para adquirir las competencias requeridas para un profesional de este siglo.

La adquisición de competencias de pensamiento crítico durante la práctica en la empresa requiere de la preparación de un cuidado plan de trabajo del estudiante que incida en la comprensión por parte del estudiante de los objetivos del trabajo que realiza, la búsqueda de información, el análisis crítico de los resultados que obtiene y la presentación y discusión de esos resultados con otros componentes del equipo.

Estas actividades de pensamiento crítico pueden organizarse de forma muy efectiva en una plantilla de cuaderno de laboratorio donde el estudiante deba recoger no solo los resultados obtenidos sino también guie las actividades de pensamiento crítico mencionadas en el párrafo anterior.

Se ha seleccionado un número reducido de capacidades de pensamiento crítico sobre las que trabajar y se ha desarrollado una metodología del trabajo en la empresa que relaciona las actividades propuestas con cada una de las capacidades que se pretende desarrollar. El cuaderno del tutor es una guía para preparar el trabajo del estudiante dirigido a desarrollar sus capacidades de pensamiento crítico. 


\section{Agradecimientos}

Este trabajo ha sido financiado por el programa Erasmus + de la dentro del proyecto 2017-1-ES01-KA202-038469, Servicio Español para la Internacionalización de la Educación (SEPIE) referencia nacional ZZ02

\section{Referencias}

DELORS, J ; AL MUFTI, I ; AMAGI, I ; CARNEIRO, R ; CHIUNG, F ; GEREMEK, B ; GORHAM, W ; KORNHAUSER, A ; MANLEY, M ; PADRON QUERO, M ; SAVANE, M-A; SINGH, K; STAVENHAGEN, R; WON SUHR, M; NANZHAO, Z (1996). Learning: The Treasure Within: Report to UNESCO of the International Commission on Education for the Twenty-First Century. Paris, UNESCO Publishing. https://unesdoc.unesco.org/ark:/48223/pf0000109590 [consulta 3 de marzo de 2019]

ENNIS R (2011). "Critical Thinking: Reflection and Perspective Part I ", Inquiry: Critical Thinking Across the Disciplines, 26 (1) : 4-37

IKASIA TECNOLOGIES SL. Proyectos http://www.ikasia.es [Consulta 21 de marzo de 2019]

LOPEZ AYMES G (2012). «Pensamiento Crítico en el Aula ». Docencia e Investigación XXXVII (22): 41-60

PAUL R, ELDER L (2005). Critical Thinking Competency Standards. Standards, Principles, Performance Indicators, and Outcomes With a Critical Thinking Master Rubric. Foundation for Critical Thinking, http://www.criticalthinking.org/ [Consulta 3 de marzo de 2019]

PITHERS RT (1999). Boosting 'Good' Thinking in Vocational Education and Training, Faculty of Education, University of Technology, Sydney, Australia, http://www.leeds.ac.uk/educol/documents/000001054.htm [Consulta 11 de marzo de 2019]

SCOTT, CL (2015). The Futures of Learning 2: What Kind of Learning for the 21st Century? EDUCATION RESEARCH AND FORESIGH 14 November 2015 United Nations Educational, Scientific and Cultural Organization working papers https://unesdoc.unesco.org/ark:/48223/pf0000242996 [Consulta 3 de marzo de 2019] 